\title{
Ethnobotanical and Economic Observations of Some Plant Resources from the Northern Parts of Pakistan
}

\author{
Hazrat Sher, Mohammad Elyemeni, Kamran \\ Hussain and Hassan Sher
}

\section{Research}

\begin{abstract}
A study on the economic value of plants being extracted from a coniferous forest of the Miandam valley of District Swat was conducted during Spring and Summer 2008. The aim of the study was to identify non-timber forest product plants being used from the target area, to identify the trade network that they are sold into, and to evaluate their value as they move within that network in order to make recommendations for socio-economic development of the area. Information was learned through semi-structured questionnaires and participatory interviews with resource uses and traders. 214 species in 79 families were identified as being useful in traditional livelihood. Of these, 150 were herbs, 36 trees, 26 shrubs and 4 climbers. Plant species are locally used as medicinal (115), multipurpose (35), fodder (31), fuel (30), vegetables (12), tools (12), timber (9), poisons (7), roof thatch (7), wild fruits (6), fences/hedges (5), veterinary (5), mud supporter (5), foods (4), spices/condiments (4), religious (4), honey bee (3), brooms (3), and evil repellent (1). Twenty out of 115 medicinal species are collected to sell. The gatherers have very little marketing skills and are often not aware of the high market value. As a result, most of collected materials are sold to local middlemen at low prices.

The study revealed that the availability of important medicinal and aromatic plant species is decreasing and the number of rare and threatened species among the medicinal and aromatic plants is increasing. Further study is, therefore, required to quantify the availability of species and to suggest suitable method for their production and conservation. Recommendations are, therefore, given in the spheres of training in identification, sustainable collection, value addition, trade monitoring and cooperative system of marketing.
\end{abstract}

\section{Introduction}

Miandam is located in the north east of district Swat and lies between $34^{\circ} 34^{\prime \prime}$ and $35^{\circ}-07^{\prime \prime} \mathrm{N}$ latitude, and $72^{\circ} 36^{\prime \prime}$ and $73^{\circ} 35^{\prime \prime} E$ longitude in the Hindu Kush mountain range. The elevation of the valley ranges from $3000 \mathrm{ft}$ to 14,000 $\mathrm{ft}$. The total area of the valley is about 36,768 acres. The valley is comprised of 11 big villages and 15 small hamlets with a population of about 20,000 people. The population of the area is mostly dependent upon agriculture and livestock rearing. About 1600 people of the total population are gathering different forest resources including medicinal and aromatic plant (MAP) species in the valley.

Pakistan hosts about 6000 species of flowering plants, out of which about 2000 species have medicinal, aromatic and economic value (Williams \& Ahmad 1999). The MAP species have supported livelihood of many people in District Swat. They are being collected for export both to national and international markets. The high dependency of

\section{Correspondence}

Hazrat Sher, Department of Botany, Kuhat University of Science and Technology, Kuhat, PAKISTAN.

Mohammad Elyemeni and Hassan Sher, Department of Botany \& Microbiology, King Saud University, P.O. Box 2455, Riyadh 11451, Riyadh, SAUDI ARABIA.

hassan.botany@gmail.com / hassansher_2000@yahoo.com

Kamran Hussain, World Wildlife Fund-Pakistan, 34-D-2 Sahibzada Abdul Qayyum Road, University Town Peshawar, PAKISTAN.

Ethnobotany Research \& Applications 9:027-041 (2011)

Published: February 08, 2011

www.ethnobotanyjournal.org/vol9/i1547-3465-09-027.pdf 
rural people on MAPs for subsistence and unsustainable harvesting practices has resulted over exploitation of resources in different sites of the study area. (Sher et al. 2005,2010 .) Similarly, conversion of natural habitats into agricultural land has greatly decreased the population size of many economically important forest resources including MAPs in different sites of the study area.

The traditional practice of extracting forest resources especially MAPs appears to be unsustainable. There is not evidence of resource tenure and custodianship, understanding of sustainable use and management parameters, harvesting and collection procedures and knowledge of market requirements. These are important barriers to the sustainable utilization of MAPs. Besides scientific understanding of population sizes, distributions, availability and abundance of plant species and their interactions with different stochastic environmental factors all play important roles in determining ecological sustainability of these resources. Similarly, quantitative and economic analysis of the effect of extraction on these ecological processes is very important to assess the effect of harvesting on development of the entire forest resources in general and MAPs in particular in order to formulate sustainable harvesting regimes.

Harvesting practices used by untrained collectors may endanger the recovery of some plants populations. Lack of knowledge about the part used, time of collection and economic value of MAP species has led to their mismanagement and least profitable exploitation. The appropriate timing to collect the desired parts of plants of certain age will determine the yield percentage and quality of therapeutically active biochemical ingredients. Gathering and processing of MAPs for family use in human and livestock treatment is a widespread, centuries old practice (Hussain et al. 2004). The use of traditional medicine for maintenance of health in most developing countries has been widely observed as a custom. Their collection and sale is an important economic activity in the northern parts of Pakistan and about 5000 families are involved in the collection and processing of MAPs in the region (Olsen \& Larsen 2003). The most active members of plant gathering and processing are women and children from middle hill areas. These collectors receive minimum wages in the trade chain of medicinal herbs (Sher et al. 2005). Therefore, the local people are losing the preservation of traditional knowledge of medicinal plants and other important non-timber forest products. The present endeavour was initiated with the aim to identify non-timber forest product plants being used from the area (coniferous forests of Miandam valley, District Swat), to identify the trade network that they are sold into, and to evaluate their value as they move within that network in order to make recommendations for socio-economic development of the area.

\section{Materials and Methods}

A study on the ethnobotanical and economic evaluation of coniferous forest of the Miandam Valley of District Swat was conducted during Spring and Summer 2008. Prior to exploring of the forest resources, topo-sheet, map and other general information of the investigated area were obtained from the Forest Department, Swat. The area was accordingly divided into different sites and then visits were made first in April and May, second in June and July, and third in August and September, 2008.

A questionnaire was prepared and used as a tool for the collection of information. The questionnaire was divided into two parts: demographics and ethnobotanical data. The first part included demographic data, location name, age, education and profession. The second part included questions about the pattern of forest resources being utilization.

\section{Field Survey}

Prior to a visit to the research sites, the questionnaire was designed and pre-tested to find out if it actually worked. Revisions needed as a result of this pre-test were noted and undertaken in the following day of the visit.

Key informants were identified from each of 210 households determined to be present in the villages in the valley. Focus groups were held with key informants and others in each household. The traditional uses (including medicinal and other use) of plant resources were learned with both the questionnaire and through participatory techniques. Participation focused on learning how people were gathering plant materials. Informants were asked about their interest as local user of plants, collectors and traders of forest resources.

Information on the market value of the plants was collected from local collectors, hakims and shopkeepers. A simple procedure was adopted asking how, from whom, and to whom the plants were sold. Respondents were also asked about their annual revenues earned from the sale of the plants and their return on work invested. For each plant species the informants mentioned, they were also asked about its abundance, distribution and population size. This was judged by comparing 20 year old records with the current situation. In addition personal observations were made in the field to note any pertinent events which could help explain the presence, and relative abundance based on the ecological characteristics of the species. The effect of current harvest on the status of each commercially valuable plant species was also studied by comparing 20 years old records with respect to the present population size and status. The effect of current harvest on the population size was finally judged based on 


\section{Sher et al. - Ethnobotanical and Economic Observations of Some Plant Resources from the Northern Parts of Pakistan}

the distance that local collectors traveled in the past as compared to the present.

Voucher specimens of the plants identified by the informants were collected from different sites of the studied valley and were dried and preserved. The fully dried specimens were mounted on herbarium paper identified with the help of available literature (Nasir \& Ali 1971-91, Stewart 1972) and deposited in National Agriculture Research Centre, herbarium, Islamabad, (RAW), and University of Karachi, Botany Department, Sindh, Pakistan (KUH).

\section{Results}

\section{Informants}

160 households were contacted out of 210 possible. Generally, the respondents were elderly persons and their age group varied from 40 to 60 years.

\section{Ethnobotanical uses}

The flora of the study area provides useful species of ethnobotanical importance. The study documented 214 plant species belonging to 79 families (Table 1). They include 148 herbs, 36 trees, 26 shrubs and 4 climbers. Plant species are locally used as medicinal (115), multipurpose (35), fodder (31), fuel (30), vegetables (12), tools (12), timber (9), poisons (7), roof thatch (7), wild fruits (6), fences/hedges (5), veterinary (5), mud supporter (5), foods (4), spices/condiments (4), religious (4), honey bee (3), brooms (3), and evil repellent (1).

\section{Ethnomedicinal uses}

The study showed that the local people have knowledge about distribution, abundance, harvesting, uses and marketing of MAPs. Plants used medicinally were grouped into 12 sub-use categories according to specific disease treatment (Table 1). The highest numbers of plants were

Table 1. Plants found to be of economic value in th coniferous forest of the Miandam Valley of District Swat, northern Pakistan. Key to Major Use Categories (Evil: Evil repellent plants; Fen: Fences/hedges; Fod: Fodder plants; Foo: Edible food; Fru: Fruits; Fue: Fuel wood; Hon: Honey bee plants; Med: Medicinal plants; MS: Mud supporter; Poi: Poisonous plants; Roo: Roof thatching; Spi: Spices/condiments; Tim: Timber wood; Too: Tool making plants; Veg: Vegetables).

\begin{tabular}{|c|c|c|c|c|}
\hline Scientific name & Family & $\begin{array}{l}\text { Vernacular } \\
\text { name(s) }\end{array}$ & $\begin{array}{l}\text { Major Use } \\
\text { Category }\end{array}$ & $\begin{array}{l}\text { Life } \\
\text { Form }\end{array}$ \\
\hline Abies pindrow (Royle ex D. Don) Royle & Pinaceae & Achar & Tim, Fue & Tree \\
\hline Alnus nitida (Spach) Endl. & Betulaceae & Gerray & Fue & Tree \\
\hline Achillea millefolium $\mathrm{L}$. & Asteraceae & Jarai, Aqarqarha & Med & Herb \\
\hline Achyranthes aspera L. & Amaranthaceae & $\begin{array}{l}\text { Charchata, } \\
\text { Largakhay }\end{array}$ & Fod & Herb \\
\hline Aconitum violaceum Jacquem. ex Stapf & Ranunculaceae & $\begin{array}{l}\text { Zaharmora, } \\
\text { Jadwar, Daghra } \\
\text { zahar }\end{array}$ & Med & Herb \\
\hline Aconitum laeve Royle & Ranunculaceae & $\begin{array}{l}\text { Zaharmora, } \\
\text { Jadwar, Daghra } \\
\text { zahar }\end{array}$ & Med & Herb \\
\hline Acorus calamus L. & Acoraceae & Skha waja & Med & Herb \\
\hline Adhatoda vasica Nees & Acanthaceae & Bekar, Bansa & Med & Herb \\
\hline Adiantum capillus-veneris L. & Pteridaceae & \begin{tabular}{|l} 
Sumbal, \\
Parsiaushah
\end{tabular} & Med & Herb \\
\hline Adiantum incisum Forssk. & Pteridaceae & Masle sumbul & Med & Herb \\
\hline Adiantum venustum D. Don & Pteridaceae & Sumbal & Med & Herb \\
\hline Aesculus indica (Wall. ex Cambess.) Hook. & Sapindaceae & Jawaz, Qatil & Fue, Med & Tree \\
\hline Ailanthus altissima (Mill). Swingle & Simaroubaceae & Bakyarna & Med, Fue & Tree \\
\hline Ajuga bracteosa Wall. ex Benth. & Lamiaceae & Booti, Neel Kanti & Med & Herb \\
\hline Ajuga parviflora Benth. & Lamiaceae & Booti & Med & Herb \\
\hline Allium cepa L. & Amaryllidaceae & Piaz & Veg & Herb \\
\hline Allium humile Kunth & Amaryllidaceae & Da ghra Ouga & Med & Herb \\
\hline
\end{tabular}




\begin{tabular}{|c|c|c|c|c|}
\hline Scientific name & Family & \begin{tabular}{|l|} 
Vernacular \\
name(s)
\end{tabular} & $\begin{array}{l}\text { Major Use } \\
\text { Category }\end{array}$ & \begin{tabular}{|l|} 
Life \\
Form \\
\end{tabular} \\
\hline Allium jacquemontii Kunth & Amaryllidaceae & Da ghra Ouga & Med & Herb \\
\hline Allium sativum $\mathrm{L}$. & Amaryllidaceae & Ougakai & Med & Herb \\
\hline Amaranthus spinosus L. & Amaranthaceae & $\begin{array}{l}\text { Da Ghany } \\
\text { Chalvaray }\end{array}$ & Fod & Herb \\
\hline Amaranthus viridis $\mathrm{L}$. & Amaranthaceae & Chalvaray & Fod, Veg & Herb \\
\hline Aquilegia fragrans Benth. & Ranunculaceae & Samer parna & Med & Herb \\
\hline Arenaria griffithii Boiss. & Caryophyllaceae & Kinar & MS, Roo & Herb \\
\hline Arisaema flavum (Forssk.) Schott & Araceae & Marjarey & Med & Herb \\
\hline Arisaema jacquemontii Blume & Araceae & Margarai & Pois & Herb \\
\hline Artemisia brevifolia Wall. ex DC. & Asteraceae & Tarkha & Med & Herb \\
\hline Artemisia scoparia Waldst. \& Kit. & Asteraceae & Dada tarkha & Bro & Herb \\
\hline Artemisia vulgaris $\mathrm{L}$. & Asteraceae & Tarkha, Afsanthen & Bro & Herb \\
\hline Asparagus adscendens Roxb. & Asparagaceae & Tindorai & Med & Shrub \\
\hline Asphodelus tenuifolius Cav. & Xanthorrhoeaceae & Ougakai & Med & Herb \\
\hline Atropa acuminata Royle ex Lindl. & Solanaceae & Bargak & Med & Herb \\
\hline Avena sativa $\mathrm{L}$. & Poaceae & Jamdaray & Med, Fod & Herb \\
\hline Berberis lycium Royle & Berberidaceae & Spin Kwaray & Med, Fue & Shrub \\
\hline Berberis vulgaris $\mathrm{L}$. & Berberidaceae & Tour Kwaray & Med, Fue & Shrub \\
\hline Bergenia ciliata (Haw.) Sternb. & Saxifragaceae & $\begin{array}{l}\text { Ghat Pana, } \\
\text { Bogandi }\end{array}$ & Med & Herb \\
\hline Bergenia stracheyi (Hook. f. \& Thomson) Engl. & Saxifragaceae & Spinsar Ghat Pana & Med & Herb \\
\hline Bistorta amplexicaulis (D. Don) Greene & Polygonaceae & $\begin{array}{l}\text { Tarwa Pana, } \\
\text { Anjabar }\end{array}$ & Med & Herb \\
\hline Bunium persicum B. Fedstch. & Apiaceae & Zankai & Spi & Herb \\
\hline Bupleurum longicaule Wall. ex DC. & Apiaceae & Gillo & Med & Herb \\
\hline Butea frondosa K.D. Koenig ex Roxb. & Fabaceae & $\begin{array}{l}\text { Da pali gul, } \\
\text { Goli tisu }\end{array}$ & Fod, Med & Herb \\
\hline Calotropis procera (Aiton) W.T. Aiton & Apocynaceae & $\begin{array}{l}\text { Goli Madar, Goli } \\
\text { akk, Spalmai }\end{array}$ & Med & Shrub \\
\hline Caltha alba Cambess. & Ranunculaceae & Makan path & Med, Veg & Herb \\
\hline Cannabis sativa $\mathrm{L}$. & Cannabaceae & Bang & Med & Herb \\
\hline Capsella bursa-pastoris (L.) Medik. & Brassicaceae & Bambesa & Med & Herb \\
\hline Caralluma edulis (Edgew.) Benth. ex Hook. f. & Apocynaceae & Pamankai & Veg & Herb \\
\hline Carum carvi $\mathrm{L}$. & Apiaceae & Zankee & Spi & Herb \\
\hline Cedrus deodara (Roxb. ex D. Don) G. Don & Pinaceae & Diar & $\begin{array}{l}\text { Tim, Fue, } \\
\text { Med }\end{array}$ & Tree \\
\hline Celtis australis L. & Cannabaceae & Tagha & Fue, Rel & Tree \\
\hline Caesalpinia decapetala (Roth) Alston & Fabaceae & Jara & Fen & Shrub \\
\hline Chamomilla recutita (L.) Rauschert. & Asteraceae & Babona & Med & Herb \\
\hline Chenopodium album $\mathrm{L}$. & Amaranthaceae & Sarmay & Med, Veg & Herb \\
\hline Chenopodium botrys L. & Amaranthaceae & Skha Kharawa & Pois & Herb \\
\hline Cichorium intybus L. & Asteraceae & Han/ Kasni & Med, Veg & Herb \\
\hline
\end{tabular}


Sher et al. - Ethnobotanical and Economic Observations of Some Plant

Resources from the Northern Parts of Pakistan

\begin{tabular}{|c|c|c|c|c|}
\hline Scientific name & Family & \begin{tabular}{|l|} 
Vernacular \\
name(s)
\end{tabular} & $\begin{array}{l}\text { Major Use } \\
\text { Category }\end{array}$ & $\begin{array}{l}\text { Life } \\
\text { Form }\end{array}$ \\
\hline Citrullus colocynthis (L.) Schrad. & Curcurbitaceae & Hunzal, Tuma & Med & Herb \\
\hline Colchicum autumnale L. & Colchicaceae & $\begin{array}{l}\text { Sarba Zeala, } \\
\text { Atees, Suranjan } \\
\text { Sherin }\end{array}$ & Med & Herb \\
\hline Colchicum luteum Baker & Colchicaceae & \begin{tabular}{|l|} 
Qaimat guallay \\
Suranjan-e-Talkh
\end{tabular} & Med & Herb \\
\hline Commelina benghalensis $\mathrm{L}$. & Commelinaceae & Narray & $\begin{array}{l}\text { Med, Fod, } \\
\text { Veg }\end{array}$ & Herb \\
\hline Corydalis govaniana Wall. & Papaveraceae & \begin{tabular}{|l|} 
Desi Mamera \\
\end{tabular} & Med & Herb \\
\hline Corydalis stewartii Fedde & Papaveraceae & Mamera & Med & Herb \\
\hline Corylus jacquemontii Decne. & Betulaceae & Zangali Badam & Med & Tree \\
\hline Cotoneaster microphyllus Wall. ex Lindl. & Rosaceae & Kharawa & Med & Shrub \\
\hline Crataegus curvisepala Lindm. & Rosaceae & Thampas & Med & Shrub \\
\hline Cuscuta reflexa Roxb. & Convolvulaceae & $\begin{array}{l}\text { Zalai, Aptemum, } \\
\text { Akash beel }\end{array}$ & Med & Climber \\
\hline Cynodon dactylon (L.) Pers. & Poaceae & Kabal & Fod & Herb \\
\hline Cynoglossum lanceolatum Forssk. & Boraginaceae & Gat gul & Med & Herb \\
\hline Cyperus brevifolius (Rottb.) Endl. ex Hassk. & Cyperaceae & $\begin{array}{l}\text { Taryela, nagar } \\
\text { motha, saad kopi }\end{array}$ & Fue & Herb \\
\hline Daphne mucronata Royle, & Thymelaeaceae & Laiughunai & Fue, Fen & Shrub \\
\hline Datura inoxia Mill. & Solanaceae & Jesmasal & Med & Herb \\
\hline Datura stramonium L. & Solanaceae & $\begin{array}{l}\text { Dahthora, } \\
\text { Harhnada }\end{array}$ & Med & Herb \\
\hline Debregeasia saeneb (Forssk.) Hepper \& Wood & Urticaceae & Ajlai & Med, Fue & Shrub \\
\hline $\begin{array}{l}\text { Delphinium denudatum Wall. ex Hook. f. \& } \\
\text { Thomson }\end{array}$ & Ranunculaceae & Jadwar & Med & Herb \\
\hline Dioscorea deltoidea Wall. ex Griseb. & Dioscoreaceae & Kaneez & Med & Climber \\
\hline Diospyros kaki Thunb. & Ebenaceae & Sur Amloke & Fru, Fue & Tree \\
\hline Diospyros lotus L. & Ebenaceae & Toor amlok & Fru, Fue & Tree \\
\hline Dryopteris filix-mas (L.) Schott & Dryopteridaceae & Ladorey & Veg & Herb \\
\hline Dryopteris juxtaposita H. Christ & Dryopteridaceae & Kwanjey & Veg & Herb \\
\hline Echinops echinatus Roxb. & Asteraceae & Manzari mangual & Med, & Herb \\
\hline Elaeagnus angustifolia L. & Elaeagnaceae & Ghanum ranga & Med, Fen & Shrub \\
\hline Equisetum arvense L. & Equisetaceae & Bandakai & Med & Herb \\
\hline Eruca sativa Mill. & Brassicaceae & \begin{tabular}{|l} 
Jawawa \\
(Taramera)
\end{tabular} & Veg & Herb \\
\hline Euphorbia helioscopia L. & Euphorbiaceae & Mandanro & Poi & Herb \\
\hline Euphorbia wallichii Hook. f. & Euphorbiaceae & Shangla & Poi & Herb \\
\hline Fagonia arabica L. & Zygophyllaceae & $\begin{array}{l}\text { Azghakai, } \\
\text { Dhamasa } \\
\end{array}$ & Med & Herb \\
\hline Ficus carica L. & Moraceae & Inzer & $\begin{array}{l}\text { Fru, Med, } \\
\text { Rel }\end{array}$ & Tree \\
\hline Ficus palmata Forssk. & Moraceae & Inzer & Fru & Tree \\
\hline
\end{tabular}




\begin{tabular}{|c|c|c|c|c|}
\hline Scientific name & Family & \begin{tabular}{|l|} 
Vernacular \\
name(s)
\end{tabular} & $\begin{array}{l}\text { Major Use } \\
\text { Category }\end{array}$ & $\begin{array}{l}\text { Life } \\
\text { Form }\end{array}$ \\
\hline Foeniculum vulgare Mill. & Apiaceae & $\begin{array}{l}\text { Kaga valaney, } \\
\text { Sonf }\end{array}$ & Med & Herb \\
\hline Fragaria vesca L. & Rosaceae & Da Zmaki toot & Fru & Herb \\
\hline Fumaria indica Pugsley & Papaveraceae & Papra, Shahtara & Med & Herb \\
\hline Geranium wallichianum D. Don ex Sweet & Geraniaceae & $\begin{array}{l}\text { Srazela, } \\
\text { Ratanjothe }\end{array}$ & Med & Herb \\
\hline Gentiana kurroo Royle & Gentianaceae & Gensing & Med & Herb \\
\hline Gynandriris sisyrinchium Parl. & Iridaceae & Cabdichar & Med & Herb \\
\hline Hedera nepalensis K. Koch & Araliaceae & Prewatai & Med & Climber \\
\hline Heracleum candicans Wall. ex DC. & Apiaceae & Kadu Pana & Med & Herb \\
\hline Hyoscyamus niger L. & Solanaceae & Bargag & Med & Herb \\
\hline Hypericum oblongifolium Choisy & Hypericaceae & Shin chai & MS & Shrub \\
\hline Hypericum perforatum $\mathrm{L}$. & Hypericaceae & Shin chai & Med & Herb \\
\hline Hyssopus officinalis L. & Lamiaceae & Goli Zofa & Med & Herb \\
\hline Indigofera heterantha Wall. ex Brandis & Fabaceae & Ghwarija & Fue, Roo & Shrub \\
\hline Isodon rugosus (Wall. ex Benth.) Codd & Lamiaceae & Sperkai & Med & Shrub \\
\hline Jasminum officinale L. & Oleaceae & Rambil chambil & Med & Climber \\
\hline Juglans regia L. & Juglandaceae & Ghuz & $\begin{array}{l}\text { Fru, Tim, } \\
\text { Fue }\end{array}$ & Tree \\
\hline Juniperus communis L. & Cupressaceae & Awbeer & Fue & Tree \\
\hline Lathyrus aphaca L. & Fabaceae & Korkamanai & Fod & Herb \\
\hline $\begin{array}{l}\text { Launaea procumbens (Roxb.) Ramayya \& } \\
\text { Rajagopal }\end{array}$ & Asteraceae & Shauda pai & Med, Fod & Herb \\
\hline Lepidium sativum $\mathrm{L}$. & Brassicaceae & Halam & Med & Herb \\
\hline Lotus corniculatus L. & Fabaceae & $\begin{array}{l}\text { Fateh khana, } \\
\text { Bopali }\end{array}$ & Med, Fod & Herb \\
\hline $\begin{array}{l}\text { Malva officinalis (L.) Schimp. \& Spenn. ex } \\
\text { Schimp. \& Spenn. }\end{array}$ & Malvaceae & Panerak & Veg & Herb \\
\hline Melia azedarach L. & Meliaceae & Tora bakyana & Fue, Vet & Tree \\
\hline Mentha longifolia (L.) Huds. & Lamiaceae & Valenay & Med & Herb \\
\hline Mentha spicata L. & Lamiaceae & Podina & Med & Herb \\
\hline $\begin{array}{l}\text { Micromeria biflora (Buch.-Ham. ex D. Don) } \\
\text { Benth. }\end{array}$ & Lamiaceae & Naray shamakay & Fod, Hon & Herb \\
\hline Morchella vulgaris (Pers.) Boud. & Morchellaceae & Gujhai & Foo & Herb \\
\hline Morchella elata Fr. & Morchellaceae & Da kwar Guchhi & Foo & Herb \\
\hline Morchella esculenta (L.) Pers. & Morchellaceae & Spina Guchhi & Foo & Herb \\
\hline Morus alba L. & Moraceae & Toot (Panjakha) & $\begin{array}{l}\text { Med, Tim, } \\
\text { Fue, Fru, } \\
\text { Too }\end{array}$ & Tree \\
\hline Morus nigra L. & Moraceae & Tour Toot & $\begin{array}{l}\text { Med, Tim, } \\
\text { Fue, Fru, } \\
\text { Too }\end{array}$ & Tree \\
\hline Morus laevigata Wall. ex Brandis & Moraceae & Shah Toot & $\begin{array}{l}\text { Tim, Fue, } \\
\text { Fru ,Too }\end{array}$ & Tree \\
\hline
\end{tabular}


Sher et al. - Ethnobotanical and Economic Observations of Some Plant

Resources from the Northern Parts of Pakistan

\begin{tabular}{|c|c|c|c|c|}
\hline Scientific name & Family & \begin{tabular}{|l|} 
Vernacular \\
name(s)
\end{tabular} & \begin{tabular}{|l|} 
Major Use \\
Category
\end{tabular} & \begin{tabular}{|l} 
Life \\
Form \\
\end{tabular} \\
\hline Myrsine africana $\mathrm{L}$. & Primulaceae & \begin{tabular}{|l} 
Babrang, \\
Marurang, Manru \\
\end{tabular} & Med & Herb \\
\hline Myrtus communis L. & Myrtaceae & Barge abulanse & Med & Shrub \\
\hline Narcissus tazetta L. & Amaryllidaceae & Gulinargus & Med, & Herb \\
\hline Nasturtium officinale R. Br. & Brassicaceae & Talmera & Veg & Herb \\
\hline Neolitsea chinensis (Gamble) Chun & Lauraceae & $\begin{array}{l}\text { Pewand zeala, } \\
\text { medachob, } \\
\text { Khadang }\end{array}$ & Med, Fod & Herb \\
\hline Nepeta govaniana (Wall. ex Benth.) Benth. & Lamiaceae & Bardranjbuya & Med & Herb \\
\hline Olea ferruginea Royle & Oleaceae & Khona & Rel, Fru & Tree \\
\hline Onosma hispida Wall. ex G. Don & Boraginaceae & Kwaga abai & Med & Herb \\
\hline Origanum vulgare $\mathrm{L}$. & Lamiaceae & Shamakay & Hon & Herb \\
\hline Otostegia limbata (Benth.) Boiss. & Lamiaceae & Spin azghai & Fen & Herb \\
\hline Oxalis corniculata L. & Oxilidaceae & Tarukay & Med & Herb \\
\hline Paeonia emodi Wall. ex Royle & Paeoniaceae & Mamekh & Med & Herb \\
\hline Papaver dubium $\mathrm{L}$. & Papaveraceae & Qashqash & Med & Herb \\
\hline Periploca aphylla Decne. & Apocynaceae & Bararra & Med & Shrub \\
\hline Picea smithiana (Wall.) Boiss. & Pinaceae & Mangazai & Tim, Fue & Tree \\
\hline Peganum harmala L. & Nitrariaceae & Spalani & Evil & Herb \\
\hline Pinus roxburghii Sarg. & Pinaceae & Chir & $\begin{array}{l}\text { Fue, Tim, } \\
\text { Too }\end{array}$ & Tree \\
\hline Pinus wallichiana A.B. Jacks. & Pinaceae & Peyoach, Kail & $\begin{array}{l}\text { Fue, Tim, } \\
\text { Too }\end{array}$ & Tree \\
\hline Pistacia integerrima J.L. Stewart ex Brandis & Anacardiaceae & $\begin{array}{l}\text { Kakar singi, } \\
\text { Shanai }\end{array}$ & Med, Fue & Tree \\
\hline Plantago lanceolata L. & Plantaginaceae & Jabai & Med & Herb \\
\hline Plantago major L. & Plantaginaceae & Gwa jabai & Med & Herb \\
\hline Platanus orientalis $\mathrm{L}$. & Platanaceae & Chinar & $\begin{array}{l}\text { Tim, Fue, } \\
\text { Too }\end{array}$ & Tree \\
\hline Plectranthus rugosus Wall. ex Benth. & Lamiaceae & Sperkay & Hon, Roo & Shrub \\
\hline Podophyllum hexandrum Royle & Berberidaceae & Kakora & Med & Herb \\
\hline Polygonatum multiflorum (L.) All. & Asparagaceae & Nooreallam & Med & Herb \\
\hline Polygonatum verticillatum (L.) All. & Asparagaceae & Nooreallam & Med & Herb \\
\hline Polygonum aviculare L. & Polygonaceae & Masloon & Med & Herb \\
\hline Populus alba L. & Salicaceae & Spina Supida & Fue & Tree \\
\hline Populus nigra L. & Salicaceae & Toura Supida & Fue & Tree \\
\hline Portulaca oleracea L. & Portulacaceae & Warkharae & Veg & Herb \\
\hline Primula denticulata Sm. & Primulaceae & Mamera & Med & Herb \\
\hline Prunus persica (L.) Batsch & Rosaceae & Tangwan & $\begin{array}{l}\text { Fru, Fod, } \\
\text { Fue }\end{array}$ & Tree \\
\hline Pteridium aquilinum (L.) Kuhn. & Dennstaedtiaceae & Kwanjai & Veg & Herb \\
\hline
\end{tabular}




\begin{tabular}{|c|c|c|c|c|}
\hline Scientific name & Family & $\begin{array}{l}\text { Vernacular } \\
\text { name(s) }\end{array}$ & $\begin{array}{l}\text { Major Use } \\
\text { Category }\end{array}$ & \begin{tabular}{|l} 
Life \\
Form
\end{tabular} \\
\hline Punica granatum $\mathrm{L}$. & Lythraceae & $\begin{array}{l}\text { Nangoray, } \\
\text { Narsawai, } \\
\text { Anarpos }\end{array}$ & Fru, Med & Shrub \\
\hline Pyrus pashia Buch.-Ham. ex D. Don & Rosaceae & Shangetei & Fru & Tree \\
\hline Quercus dilatata Lindl. & Fagaceae & Toor banj & $\begin{array}{l}\text { Fue, Fod, } \\
\text { Too }\end{array}$ & Tree \\
\hline Quercus incana W. Bartram & Fagaceae & Spin banj & \begin{tabular}{|l|} 
Med, Fue, \\
Fod, Too \\
\end{tabular} & Tree \\
\hline Ranunculus arvensis $\mathrm{L}$. & Ranunculaceae & Ziar Gulay & Poi & Herb \\
\hline Ranunculus muricatus L. & Ranunculaceae & Ziar Gulay & Fod & Herb \\
\hline Rheum webbianum Royle & Polygonaceae & $\begin{array}{l}\text { Chotial, } \\
\text { Rewand chini }\end{array}$ & Med & Herb \\
\hline Rhus semialata Murray & Anacardiaceae & Samaqdana & Med & Herb \\
\hline Ricinus communis $\mathrm{L}$. & Euphorbiaceae & Harhanda & Pois & Shrub \\
\hline Rosa moschata Mill. & Rosaceae & Kurach, Gulab & Med, Fen & Shrub \\
\hline Rosa webbiana Wall. ex Royle & Rosaceae & Phalwari & Med, & Herb \\
\hline Rubia cordifolia L. & Rubiaceae & Srajarai / mangeet & Fod & Herb \\
\hline Rubus fruticosus L. & Rosaceae & Karwara & Med, Fen & Herb \\
\hline Rumex dentatus L. & Polygonaceae & Shalkhay & Veg & Herb \\
\hline Rumex hastatus D. Don, & Polygonaceae & Tarukey & Med & Herb \\
\hline Saccharum griffithii Munro ex Boiss. & Poaceae & Kahay & Too & Herb \\
\hline Saccharum spontaneum L. & Poaceae & Naray kahay & Too & Herb \\
\hline Salvia lanata Roxb. & Lamiaceae & Spera botai & Med & Herb \\
\hline Salvia moorcroftiana Wall. ex Benth. & Lamiaceae & Kherghwag & Med, Roo & Herb \\
\hline Salix alba L. & Salicaceae & Wala & Too & Tree \\
\hline Salix babylonica L. & Salicaceae & Wala & Too, Roo & Tree \\
\hline Salix tetrasperma Roxb. & Salicaceae & Wala & Too & Tree \\
\hline Sambucus wightiana Wall. ex Wight \& Arn. & Caprifoliaceae & Benakai & Fue, Roo & Herb \\
\hline Sapindus detergens Roxb. & Sapindaceae & Reta & Med, & Shrub \\
\hline Sarcococca saligna (D. Don) Müll. Arg. & Euphorbiaceae & Ladanr & Roo & Herb \\
\hline Saussurea atkinsonii C.B. Clarke & Asteraceae & Sharshamay & Med & Herb \\
\hline Saussurea lappa (Decne.) Sch. Bip. & Asteraceae & Kut & Med & Herb \\
\hline Senecio chrysanthemoides DC. & Asteraceae & Da Ghra Gul & Med & Herb \\
\hline Sesamum indicum L. & Pedaliaceae & $\begin{array}{l}\text { Tori Kowanzali, } \\
\text { til seah } \\
\end{array}$ & Med & Herb \\
\hline Silene vulgaris (Moench) Garcke & Caryophylaceae & Matorangay & Med & Herb \\
\hline Sisymbrium irio L. & Brassicaceae & $\begin{array}{l}\text { Rai, Khak Sher } \\
\text { Kamsal arway }\end{array}$ & Med & Herb \\
\hline Skimmia laureola (DC.) Siebold \& Zucc. ex Walp. & Rutaceae & Nazar Panra & Evil, Vet & Herb \\
\hline Solanum nigrum L. & Solanaceae & Kamacho, Makoha & Med, Vet & Herb \\
\hline Solanum surattense Burm. f. & Solanaceae & Manraghonay & Vet & Herb \\
\hline Solanum xanthocarpum Schrad. \& H. Wendl. & Solanaceae & Marraghonary & Poi & Herb \\
\hline
\end{tabular}


Sher et al. - Ethnobotanical and Economic Observations of Some Plant Resources from the Northern Parts of Pakistan

\begin{tabular}{|c|c|c|c|c|}
\hline Scientific name & Family & $\begin{array}{l}\text { Vernacular } \\
\text { name(s) }\end{array}$ & $\begin{array}{l}\text { Major Use } \\
\text { Category }\end{array}$ & $\begin{array}{l}\text { Life } \\
\text { Form }\end{array}$ \\
\hline Sonchus asper (L.) Hill & Asteraceae & Shauda pai & Fod, Vet & Herb \\
\hline Sorgum halepense (L.) Pers. & Poaceae & Spirkey & Fod & Herb \\
\hline Spiraea chinensis Maxim. & Rosaceae & Krachae & Roo & Herb \\
\hline Stachys parviflora Benth. & Lamiaceae & $\begin{array}{l}\text { Sper Botai, } \\
\text { Kundiyari }\end{array}$ & Pois & Herb \\
\hline Stellaria media (L.) Vill. & Caryophyllaceae & Oulalai & Fod & Herb \\
\hline Swertia alata C.B. Clarke & Gentianaceae & Chirat botay & Med & Herb \\
\hline Taraxacum officinale F.H. Wigg. & Asteraceae & Ziar guali & Fod, & Herb \\
\hline Taxus wallichiana Zucc. & Taxaceae & Banrya & Med, Fue & Tree \\
\hline Thymus serpyllum L. & Lamiaceae & $\begin{array}{l}\text { Zangali ajwain, } \\
\text { Sperkai }\end{array}$ & Med & Herb \\
\hline Trachyspermum ammi (L.) Sprague & Apiaceae & Sperkai & Spi & Herb \\
\hline Tribulus terrestris L. & Zygophylaceae & Markundai & Med & Herb \\
\hline Trifolium repens L. & Fabaceae & Shaftal & Fod & Herb \\
\hline Trigonella foenum-graecum L. & Fabaceae & Malkhwazi & Spi & Herb \\
\hline Urtica dioica L. & Urticaceae & Jalbang & Veg & Herb \\
\hline Valeriana jatamansi Jones & Caprifoliaceae & Mushk-e-Bala & Med & Herb \\
\hline Verbascum thapsus L. & Scrophulariaceae & Khardag & Med & Herb \\
\hline Verbena officinalis L. & Verbenaceae & Shamakai & Fod & Herb \\
\hline Viburnum grandiflorum Wall. ex DC. & Adoxaceae & $\begin{array}{l}\text { Amoch, Ghuz } \\
\text { mava }\end{array}$ & MS & Shrub \\
\hline Viburnum nervosum D. Don & Adoxaceae & $\begin{array}{l}\text { Amoch, Ghuz } \\
\text { mava }\end{array}$ & MS & Shrub \\
\hline Viola biflora L. & Violaceae & Banafsha & Med & Herb \\
\hline Viola serpens Wall. ex Ging. & Violaceae & Banafsha & Med & Herb \\
\hline Vitex negundo L. & Verbenaceae & Maravandai & Rel, MS & Shrub \\
\hline Withania coagulans (Stocks) Dunal & Solanaceae & $\begin{array}{l}\text { Paneer doda, } \\
\text { Shapiyanga }\end{array}$ & Med & Herb \\
\hline Withania somnifera (L.) Dunal & Solanaceae & Kotilal, Asgand & Med & Shrub \\
\hline Woodfordia fruticosa (L.) Kurz & Lythraceae & Zangali anar & Med, MS & Shrub \\
\hline Zanthoxylum armatum DC. & Rutaceae & Dambara & Med & Tree \\
\hline Ziziphus sativa Gaertn. & Rhamnaceae & Markhanai, Unnab & Fru, Fod & Tree \\
\hline Zizyphus mauritiana Lam. & Rhamnaceae & Karkana & $\begin{array}{l}\text { Fru, Roo, } \\
\text { Fod }\end{array}$ & Shrub \\
\hline Zizyphus vulgaris Lam. & Rhamnaceae & Markhanai/Beer & Fue, Fod & Tree \\
\hline
\end{tabular}

found to be used for the treatment of stomach and gastrointestinal problems (15 species). Similarly 10 species were used as general body tonic and arthritis, 10 species used for curing of skin disease, aching, scabies and eczema, 05 species were used as anti helmenthic, 7 species were used for curing of sore throat, chest pain and fever, 4 species for treating liver diseases, 6 species for the removal of kidney stone, 3 species for swelling, 2 species for cut and wound healing, 2 species for curing of nervous disorder, 2 species as laxative and 3 used for the control of diabetes.

Sustainable harvesting of the plants depends on the parts used, time and method of harvesting. Therefore, information regarding the harvesting and use of specific parts of MAPs species were also collected. Out of 115 MAP spe- 
cies, about 20 plants were used and harvested for their roots and rhizomes. Other most common plants parts were leaves (18 species) followed by other plant parts (Figure 1).

\section{Level of knowledge about plant resources}

The study revealed that the level of knowledge differs in extent among gender, occupational and social groups. For example the Hakims and tenants possess comparatively higher knowledge about MAPs than the general population. Similarly, men have more knowledge than women. Among the men elders were more aware than the younger members of the community.

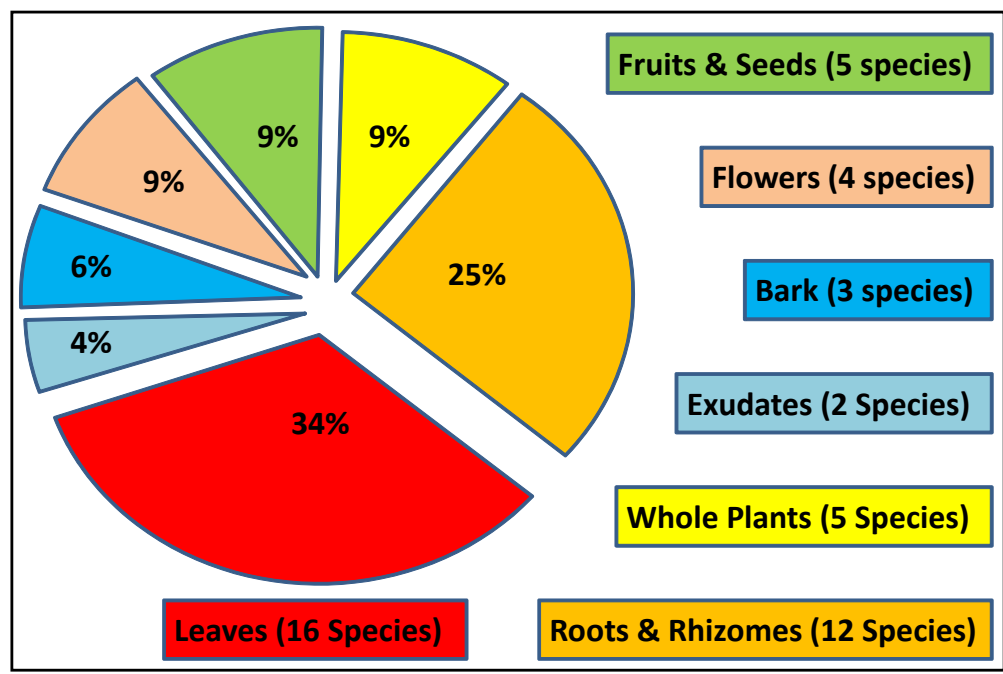

\section{Market study}

Figure 1. Medicinal and aromatic plant species by category of parts used in coniferous forest of the Miandam Valley of District Swat, northern Pakistan.

The survey revealed that the marketing of MAPs in the area is in the hands of specific collectors and few local shopkeepers. In the study area there are several regular collectors among the residents. They collect considerable quantities of MAPs and supply them to various domestic and national trading centers of Pakistan including local bazaars in Mingora, Peshawar, Islamabad, Lahore and Karachi as well as abroad. The study revealed that out of 114

reported MAPs a total twenty species (Table 2) have high market value and are collected in large amounts for sale. Among these plants the highest price is paid for Morchella esculenta (L.) Pers., Viola serpens Wall. ex Ging., Valeriana jatamansi Jones, Podophyllum hexandrum Royle, Trachyspermum ammi (L.) Sprague, and Bistorta amplexicaulis (D. Don) Greene. The prices of each species vary from year to year and also depend on demand and supply.

Table 2. Prices and production volumes for maketable medicinal plants of Miandam Valley, northern Pakistan when sold in Miandam, Madam and other markets.

\begin{tabular}{|c|c|c|c|c|c|c|c|c|}
\hline \multirow[t]{2}{*}{ Scientific Name } & \multirow{2}{*}{$\begin{array}{l}\text { Local } \\
\text { Name }\end{array}$} & \multirow[t]{2}{*}{ Part Used } & \multicolumn{3}{|c|}{ In Miandam } & \multicolumn{2}{|c|}{ In Madam } & \multirow{2}{*}{\begin{tabular}{l}
\multicolumn{1}{c}{$\begin{array}{c}\text { Oher } \\
\text { markets }\end{array}$} \\
Price / \\
Kg (Rs)
\end{tabular}} \\
\hline & & & \multicolumn{2}{|c|}{$\begin{array}{l}\text { Pricel } \\
\text { Kg (Rs) }\end{array}$} & \multirow{2}{*}{$\begin{array}{l}\text { Total } \\
\text { prod / } \\
\text { year } \\
2000 \\
\end{array}$} & \multirow{2}{*}{$\begin{array}{l}\text { Price / } \\
\text { Kg (Rs) } \\
18\end{array}$} & \multirow{2}{*}{$\begin{array}{l}\begin{array}{l}\text { Total } \\
\text { prod } / \\
\text { year }\end{array} \\
9500 \\
\end{array}$} & \\
\hline Adiantum venustum D. Don & Persusha & dried herb & 10 & 14 & & & & 40 \\
\hline $\begin{array}{l}\text { Ajuga bracteosa Wall. } \\
\text { ex Benth. }\end{array}$ & Booti & dried herb & 15 & 60 & 15 & 80 & 100 & 200 \\
\hline Allium sativum $\mathrm{L}$. & Ouga & $\begin{array}{l}\text { dried whole } \\
\text { plant }\end{array}$ & 50 & 40 & 200 & 25 & 50000 & 50 \\
\hline Berberis lycium Royle & Kwaray & $\begin{array}{l}\text { dried root } \\
\text { bark }\end{array}$ & 20 & 50 & 70 & 120 & 100 & 150 \\
\hline $\begin{array}{l}\text { Bistorta amplexicaulis (D. Don) } \\
\text { Greene }\end{array}$ & $\begin{array}{l}\text { Tarwa } \\
\text { Pana }\end{array}$ & $\begin{array}{l}\text { dried } \\
\text { rhizome }\end{array}$ & 50 & 20 & 2500 & 25 & 60000 & 40 \\
\hline Caltha alba Cambess. & $\begin{array}{l}\text { Makan } \\
\text { path }\end{array}$ & $\begin{array}{l}\text { fresh floral } \\
\text { shoot }\end{array}$ & 10 & 14 & 5000 & - & - & - \\
\hline Cichorium intybus L. & Han & fresh root & 10 & - & - & - & - & - \\
\hline Diospyros lotus L. & Toor amlok & $\begin{array}{l}\text { dried/ ripe } \\
\text { fruits }\end{array}$ & 10 & 13 & 9000 & 20 & 600000 & 30 \\
\hline
\end{tabular}


Sher et al. - Ethnobotanical and Economic Observations of Some Plant Resources from the Northern Parts of Pakistan

\begin{tabular}{|c|c|c|c|c|c|c|c|c|}
\hline \multirow[t]{2}{*}{ Scientific Name } & \multirow{2}{*}{$\begin{array}{l}\text { Local } \\
\text { Name }\end{array}$} & \multirow[t]{2}{*}{ Part Used } & \multicolumn{3}{|c|}{ In Miandam } & \multicolumn{2}{|c|}{ In Madam } & \multirow{2}{*}{\begin{tabular}{|l}
$\begin{array}{c}\text { Oher } \\
\text { markets }\end{array}$ \\
Price I \\
Kg (Rs)
\end{tabular}} \\
\hline & & & $\begin{array}{l}\text { Price } \\
\mathrm{Kg}(\mathrm{F}\end{array}$ & & \begin{tabular}{|l|} 
Total \\
prod I \\
year
\end{tabular} & $\begin{array}{l}\text { Price I } \\
\text { Kg (Rs) }\end{array}$ & $\begin{array}{l}\text { Total } \\
\text { prod I } \\
\text { year }\end{array}$ & \\
\hline Dryopteris juxtaposita H. Christ & Kwanjay & $\begin{array}{l}\text { Fresh whole } \\
\text { frond }\end{array}$ & 10 & 13 & 5000 & 15 & 1000 & 20 \\
\hline Mentha longifolia (L.) Huds. & Valenay & dried shoots & 10 & 30 & 20 & 60 & 150 & 100 \\
\hline Morchella esculenta (L.) Pers. & Gujhai & $\begin{array}{l}\text { dried whole } \\
\text { fruiting body }\end{array}$ & 300 & 5000 & 20000 & 6000 & 1000 & 11000 \\
\hline Paeonia emodi Wall. ex Royle & Mamekh & dried herb & 80 & 30 & 1000 & 40 & 7000 & 80 \\
\hline $\begin{array}{l}\text { Pistacia integerrima J.L. } \\
\text { Stewart ex Brandis }\end{array}$ & Shanai & $\begin{array}{l}\text { dried insect } \\
\text { galls, leaves } \\
\text { and bark }\end{array}$ & 80 & & & & & \\
\hline $\begin{array}{l}\text { Podophyllum hexandrum } \\
\text { Royle }\end{array}$ & Kakora & \begin{tabular}{|l} 
dried \\
rhizome
\end{tabular} & 75 & 140 & 50 & 170 & 100 & 400 \\
\hline Punica granatum $\mathrm{L}$. & Nangoray & $\begin{array}{l}\text { dried } \\
\text { capsule }\end{array}$ & 50 & & & & & \\
\hline $\begin{array}{l}\text { Skimmia laureola (DC.) } \\
\text { Siebold \& Zucc. ex Walp. }\end{array}$ & Nazar pana & fresh leaves & 20 & 20 & 1000 & 40 & 6000 & 70 \\
\hline $\begin{array}{l}\text { Trachyspermum ammi (L.) } \\
\text { Sprague }\end{array}$ & Sperkai & dried fruit & 150 & 120 & 10 & 150 & 50 & 180 \\
\hline Valeriana jatamansi Jones & $\begin{array}{l}\text { Muske } \\
\text { Bala }\end{array}$ & $\begin{array}{l}\begin{array}{l}\text { dried } \\
\text { rhizome }\end{array} \\
\end{array}$ & 200 & 120 & 1500 & 160 & 60000 & 200 \\
\hline Viola serpens Wall. ex Ging. & Banafsha & dried herb & 30 & 200 & 3000 & 270 & 70000 & 320 \\
\hline Zanthoxylum armatum DC. & Dambara & dried fruit & 100 & 60 & 20 & 90 & 100 & 120 \\
\hline
\end{tabular}

During the survey it was generally observed that for obtaining the plant materials from the study area, the dealers from national markets send representatives to local dealers in Mingora and put their demand. The local dealers pass the message to their agents, i.e., the local shopkeepers of the valley. These agents inform small shopkeepers and collectors. The collectors gather the species for the local shopkeepers and agents. The collectors are illiterate and do not negotiate for the price of the plant materials and gather sizeable quantities but do not receive reasonable returns (Table 2). The dealers of Mingora get the material from the agents when that is ready. In this way the medicinal plants pass through three or four hands (Table 2). Some times daily wages laborers working for Rs. 200-300 per day are employed by the local agents for plant collection but this is not usual.

\section{Price fluctuations of species at collector level and beyond}

The present study evaluated that the price of the plants gradually increases from collector to local, national and international markets at each step in the chain (Table 3). The price was lowest at the collectors level and increasing many fold from collectors to the national markets and abroad. However, while guessing at the increase of the price from the collector level and beyond, it may be kept in mind that considerable weight of the plant material is lost during drying, cleaning, processing, grading, packing etc. at each level when value is added to the material. This weight loss varies from species to species and the modes of processing for sale. One of the reasons for the low price at collectors' level was their lack of awareness of the price of the plants in the trade markets.

\section{Investment of work by collectors}

Time invested was based on the relative availability of the species with respect to the distance and the characteristics of the plant parts. Usually 4-8 hours work at 3-4 km distance was required to gather a bundle of roots of $40-50$ $\mathrm{kg}$ of for plants like Dioscorea deltoidea Wall. ex Griseb., $B$. amplexicaule, $P$. hexandrum, Paeonia emodi Wall. ex Royle and $V$. jatamansi. The flowers of $V$. serpens and $H y-$ pericum perforatum $\mathrm{L}$. required $6-8$ hours time at $3-4 \mathrm{~km}$ to collect one $\mathrm{kg}$ of flowers (Table 4). Each species is collected manually with high labor cost as compared to other daily activities conducted by locals so the collectors get much less money for their labor. 
Table 3. Market value chain of average prices in Pakistan rupee (PKR) Rs. per kilogram of plant material at different market points. measured from the source of medicinal plant material in Miandam Valley, northern Pakistan.

\begin{tabular}{|c|c|c|c|c|c|c|c|}
\hline \multirow[t]{3}{*}{ Scientific Name } & \multirow{3}{*}{$\begin{array}{l}\text { Parts } \\
\text { Collected }\end{array}$} & \multirow{3}{*}{$\begin{array}{l}\text { Demand } \\
\text { Form }\end{array}$} & \multicolumn{5}{|c|}{ Rates (Rs. per kg) } \\
\hline & & & \multirow{2}{*}{\begin{tabular}{|l|} 
Collectors \\
Collector
\end{tabular}} & \multicolumn{4}{|l|}{ Markets } \\
\hline & & & & $\begin{array}{l}\text { Local } \\
\text { Market }\end{array}$ & $\begin{array}{l}\text { Whole } \\
\text { Sale }\end{array}$ & National & $\begin{array}{l}\text { Inter- } \\
\text { national }\end{array}$ \\
\hline Aconitum laeve Royle & rhizomes & dried & 60 & 100 & 150 & 200 & 300 \\
\hline $\begin{array}{l}\text { Aconitum heterophyllum Wall. } \\
\text { ex Royle }\end{array}$ & rhizomes & dried & 60 & 100 & 150 & 200 & 300 \\
\hline Berberis lycium Royle & bark & dried & 40 & 60 & 80 & 150 & --- \\
\hline Berberis vulgaris $\mathrm{L}$. & bark & dried & 50 & 80 & 100 & 200 & --- \\
\hline $\begin{array}{l}\text { Bistorta amplexicaulis (D. Don) } \\
\text { Greene }\end{array}$ & rhizomes & dried & 80 & 120 & 150 & 200 & 300 \\
\hline $\begin{array}{l}\text { Dioscorea deltoidea Wall. } \\
\text { ex Griseb. }\end{array}$ & rhizomes & dried & 30 & 50 & 80 & 150 & 250 \\
\hline Morchella esculenta (L.) Pers. & $\begin{array}{l}\text { fruiting } \\
\text { bodies }\end{array}$ & fresh & 5000 & 10000 & 18000 & 25000 & 35000 \\
\hline Podophyllum hexandrum Royle & rhizomes & dried & 140 & 170 & 200 & 250 & 500 \\
\hline Paeonia emodi Wall. ex Royle & rhizomes & dried & 40 & 60 & 100 & 150 & 200 \\
\hline Viola serpens Wall. ex Ging. & $\begin{array}{l}\text { leaves \& } \\
\text { flowers }\end{array}$ & dried & 200 & 250 & 350 & 500 & 1000 \\
\hline Valeriana jatamansi Jones & rhizomes & dried & 160 & 200 & 300 & 350 & 500 \\
\hline
\end{tabular}

Table 4 indicates that the population of the selected species has been reduced by more than half in the last 20 to 30 years. The local people traveled one to two $\mathrm{km}$ for the collection of some quantity (e.g., $50 \mathrm{~kg}$ of rhizomes of $D$. deltoidea, P. hexandrum, P. emodi, V. jatamansi and $B$. amplexicaule and one $\mathrm{kg}$ flower / leaves of $\mathrm{V}$. sepens and $H$. perforatum) of the species in the past and now they traveled 2 to $4 \mathrm{~km}$ for the same quantity. Similarly they used to spend one to 4 hours 20 to 30 years ago and now they spend 3 to 8 hours for the same collections.

Table 4. Differences in work investment (distance traveled and time spent) for collection of targeted plants in Miandam Valley, northern Pakistan over a 20 year period of time.

\begin{tabular}{|c|c|c|c|c|c|}
\hline \multirow[t]{2}{*}{ Scientific Name } & \multicolumn{2}{|c|}{ Distance Traveled (km) } & \multicolumn{2}{|c|}{ Time Spent (hours) } & \multirow{2}{*}{$\begin{array}{l}\text { Form of } \\
\text { material } \\
\text { sold }\end{array}$} \\
\hline & 2008 & 1988 & 2008 & 1988 & \\
\hline Aconitum laeve Royle & 3 & 1 & $4-5$ & $2-3$ & dried \\
\hline Aconitum heterophyllum Wall. ex Royle & 3 & 1 & $4-5$ & $2-3$ & dried \\
\hline Berberis lycium Royle & 3 & 1 & $4-5$ & $2-3$ & dried \\
\hline Berberis vulgaris L. & 2 & 1 & $3-4$ & $1-2$ & dried \\
\hline $\begin{array}{l}\text { Bistorta amplexicaulis (D. Don) } \\
\text { Greene }\end{array}$ & 3 & 1 & $4-5$ & $2-3$ & dried \\
\hline $\begin{array}{l}\text { Dioscorea deltoidea Wall. } \\
\text { ex Griseb. }\end{array}$ & 3 & 1 & $4-5$ & $2-3$ & dried \\
\hline Hypericum perforatum L. & $3-4$ & 2 & $6-8$ & $3-4$ & fresh \\
\hline Morchella esculenta (L.) Pers. & 3 & 1 & $4-5$ & $2-3$ & dried \\
\hline Paeonia emodi Wall. ex Royle & 3 & 1 & $4-5$ & $2-3$ & dried \\
\hline Podophyllum hexandrum Royle & 3 & 1 & $4-5$ & $2-3$ & dried \\
\hline Valeriana jatamansi Jones & 3 & 1 & $4-5$ & $2-3$ & dried \\
\hline Viola serpens Wall. ex Ging. & $3-4$ & 2 & $6-8$ & $3-4$ & fresh \\
\hline
\end{tabular}




\section{Sher et al. - Ethnobotanical and Economic Observations of Some Plant Resources from the Northern Parts of Pakistan}

\section{Collectors involved}

The information at the local level had shown that in the study area children were the main collectors $(60 \%)$ followed by women (30\%) local men (15\%) and outsiders $(5 \%)$.

\section{Holy and religious plants}

Table 1 shows that a total of six species, namely Celtis australis L., Ficus carica L., Olea ferruginea Royle and Vitex negundo L. were considered holy or religious trees. They were mostly grown in holy and sacred places such as in shrines, mosques or grave yards.

\section{Tool making species}

Twelve species, including Morus nigra L., Morus alba L., O. ferruginea, Saccharum griffithii Munro ex Boiss., Saccharum spontaneum L. and Quercus dilatata Lindl. were utilized in toll making purposes like plough, digger handle, axles handles, cart handles, and water wheel. Writing pens were made from $S$. griffithii and $S$. spontanum. The needles of Pinus roxburgii Sarg. and Pinus wallichiana A.B. Jacks. are used for fruit packing. Similarly, $P$. roxburgii leaves and the stems of $S$. griffithii and $S$. spontanum are used for making brooms (Table 1).

\section{Fuel wood}

A total of 30 species (Table 1) are used for fuel wood. All of the human population in district Swat is dependant on the forests for fuel wood requirements so these plants are essential.

\section{Fodder}

Twenty-eight species are used as fodder. Fodder is drawn from forest, pasture and rangeland.

\section{Vegetables and Wild Fruits}

Twelve wild plants are collected and used as vegetables. Most of these are consumed locally, because of low quantities, higher cost of collection and low prices. Six wild fruit species are gathered with very small quantities marketed.

\section{Discussion}

The study identifies that the people of the area rely on 214 plants for medicines, food, fuel, fodder, building materials and other daily products. In spite of the economic importance of any of these species, it is unclear if any are either locally endangered or appears to be on the verge of becoming locally extinct. FAO, (1995) reported the major links between forestry and food security viz: environment, production and socio-economic linkages, which are interrelated. Therefore, forestation with the participation of lo- cal population could play a useful role in raising the living standard of these rural people as forests are just one element within the complex fabric of rural life.

The natural vegetation of the study area is under heavy pressure as a result of overgrazing, illicit cutting, and unauthorized collection of MAPs. Although, plants have varied ethnobotanical uses, they may not be well managed. Olsen and Larsen (2003) and Hussain et al. (2004) reported that the number of endangered species is increasing due to environmental degradation, over grazing and over exploitation in the form of medicinal plants extraction and deforestation.

Most of the reported species have multiple uses and these are invariably used for treating local health care needs and for earning extra income. McDicken and Mehl (1990), besides highlighting the improvement aspect in multipurpose trees, also touched upon fruit and fodder aspects of these species. The present study revealed that multipurpose trees and other woody perennials are important sources of food and income generation for rural households. In addition to these advantages, some species have other uses such as fodder, fuel wood, timber wood and medicine. Gunasena (1994) reported that in Sri Lanka, fruit trees (e.g., break fruit, Artocarpus altilis (Parkinson) Fosberg) were supplementing or substituting for the staple rice diet. In Swat several species of fruit trees may serve as a genetic resource base for improvement of commercial species. Some are already used as root stock for grafting/budding of commercial fruit varieties. Exchanging of germplasm in these fruit trees having a wider range of distribution would be very useful. Examples are: Juglans regia L., Diospyros lotus L., F. carica, Pyrus communis L., $M$. alba and $M$. nigra. There is a need to conserve these resources for the future, as they are refuges for valuable and endangered animals, especially birds, as well as for our own survival.

Most of the species identified from Swat are marketable and provide the opportunity to supplement household income. The market information revealed that district Swat is the collection and trading center for many MAPs. It has a well-established market which supplies sizeable quantities of MAPs to various trading centers of Pakistan and abroad. Sher et al. (2005) also reported that majority of marketable MAPs are collected from Northern areas of Pakistan including Swat. The present study noticed that the structure of MAP trade is complex involving many players. The middlemen who purchase material from collectors sell them either to small shops in the region or to regional middlemen or agents of large dealers, and through this chain of middlemen the material reaches the wholesale dealers of large cities. Wholesalers supply the plant materials then to retailers or pharmaceutical companies or exporters. The price may go quite high as the materials move from the collectors to international markets. The present study observed that prices increase 3 to 5 
fold from collectors to local dealers. However, income derived from the sale of wild plant species is of particular importance to the poorer households who must supplement food production with cash in order to meet basic needs.

It was also observed that the MAP species are collected from the wild of the study area on what is apparently a first-come, first-serve basis. There is no management structure tied to the harvesting of resources at present. Therefore, collection volume is likely beyond the regeneration rate. Similar observations were also reported by Sher et al. (2010) and Sher et al. (2005). They stated that the trade and collection of plant materials is mostly handled by unskilled persons. As a result valuable MAPs are damaged due to lack of scientific methods of collection. It was also discovered that collectors now have to exert greater effort and to walk longer distances to collect the same materials of MAPs when compared to twenty years ago. We recorded an increase in elevation and remoteness of the area within the Swat valley where plants are collected, involvement of children and women in the collection of, and dependence on MAPs increased. This agrees with the studies of Khan (1998) who reported that women and children usually gather medicinal plants as a part time business, in the northern areas of Pakistan.

\section{Conclusions and Recommendations}

The area has endemic and endangered plant species, some of which are of medicinal and economic importance. Indigenous knowledge behind their uses, collection and management is eroding. One reason for this is the lack of awareness among the local community regarding the economic and medicinal importance of MAPs. Another factor contributing in the decline of availability MAPs in the area and eroding of indigenous knowledge is the inadequacy of the MAPs market and lack of government support for it. This is, therefore, an issue of national policies and must be addressed.

The study recorded some valuable information about specific MAP species that merits further inquiry. For instance, Rheum webbianum Royle is widely distributed and quite common in Swat valley. Chinese scientists have developed small-scale enterprises for species like this including preparation of jam and prickles, etc. Rheum webbanium could be developed similarly in our country.

The population cover and potential density of MAPs appears to be rapidly decreasing. We call for development of adequate size in-situ conservation plots for better management of MAP species. One important lesson learned from this study is that the establishment of a community based enterprise that depends on local biodiversity can be a strategy to provide more equitable returns to community groups and hence incentives for conserving the resource base. In order to ensure the management and conservation of MAPs, documenting of indigenous knowledge systems and their constant and consistent support is essential for success of conservation in Swat.

\section{Acknowledgements}

We greatly thank the local communities for their willingness to sharing their valuable empirical knowledge and experiences on wild medicinal plants. Thanks are also due to the Forest Department for secondary data of the study area.

\section{Literature Cited}

FAO. 1995. Forest Resources Assessment 1990 - Global synthesis. Food and Agriculture Organization, Forestry Paper No. 124. FAO, Rome. www.fao.org/docrep/007/ v5695e/v5695e00.htm

Gunasena, H.P.M. 1994. Conservation and Utilization of Multipurpose Trees for Rural Development and Food Security. Paper presented at International Seminar on Conservation/Utilization of MTS for Rural Development, Taiwan Forest Research Institute, Taipei.

Hussain, F., H. Sher \& M. Ibrar. 2004. Ethnomedicinal profile of some plants of district Swat, Pakistan. Pakistan Journal of Plant Sciences 10(2):85-104.

Khan, M.H. 1998. Biodiversity of medicinal and economic plants in Northern Himalayan region, Azad Kashmir. Pp. 6-10 in Proceedings of Wild Plant Resources of Northern Pakistan, Workshop. Pakistan Forest Institute, Peshawar, Northwest Frontier Province, Pakistan. May 11-12, 1998.

McDicken, K.G \& C.B. Mehl. 1990. Farmer perspectives on improvement objectives of MPTs. Pp. 56-60 in Tree Improvement of Multipurpose Species. Edited by N. Glover \& N. Adams. Multipurpose Tree Species Network Technical Series, Volume 2. Winrock International, Washington, D.C.

Nasir, E. \& S.I. Ali. 1971-1994. Flora of Pakistan. Pakistan Agriculture Research Council, Islamabad.

Olsen, C.S. \& H.O. Larsen. 2003. Alpine medicinal plant trade and and Himalayan mountain livelihood strategies. The Geographical Journal 169(3):243-254.

Sher. H., F. Hussain \& H. Sher. 2010. Ex-situ management study of some high value medicinal plant species in Swat, Pakistan. Ethnobotany Research and Applications 8:17-24.

Sher, H. Z.D. Khan, A.U. Khan \& F. Hussain. 2005. In-situ protection management and conservation study of some medicinal plants. Acta Botanica Yunnanica 27:27-36. 


\section{Sher et al. - Ethnobotanical and Economic Observations of Some Plant Resources from the Northern Parts of Pakistan}

Stewart. R.R. 1972. An Annotated Catalogue of the Vascular Plants of West Pakistan and Kashmir. Farkhri Press Karachi.

Williams, J.T. \& J. Ahmad. 1999. Priorities for Medicinal plants. Research and Development in Pakistan. Medici- nal and Aromatic Plants Program in Asia (MAPPA). International Development Research Centre (IDRC), Canada, Medicinal and Aromatic Plants Program in Asia-SARO, New Delhi. 
